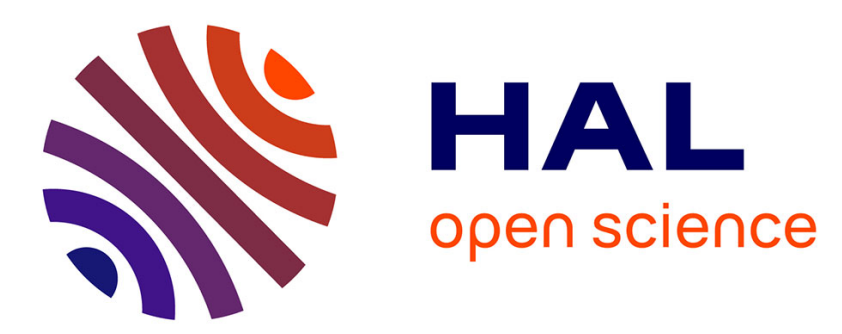

\title{
On the derivation of thermomechanical balance equations for continuous systems with a nonmaterial interface
}

Francesco Dell'Isola, A. Romano

\section{- To cite this version:}

Francesco Dell'Isola, A. Romano. On the derivation of thermomechanical balance equations for continuous systems with a nonmaterial interface. International Journal of Engineering Science, 1987, pp.10. hal-00502483

\section{HAL Id: hal-00502483 \\ https://hal.science/hal-00502483}

Submitted on 15 Jul 2010

HAL is a multi-disciplinary open access archive for the deposit and dissemination of scientific research documents, whether they are published or not. The documents may come from teaching and research institutions in France or abroad, or from public or private research centers.
L'archive ouverte pluridisciplinaire HAL, est destinée au dépôt et à la diffusion de documents scientifiques de niveau recherche, publiés ou non, émanant des établissements d'enseignement et de recherche français ou étrangers, des laboratoires publics ou privés. 


\title{
ON THE DERIVATION OF THERMOMECHANICAL BALANCE EQUATIONS FOR CONTINUOUS SYSTEMS WITH A NONMATERIAL INTERFACE $\uparrow$
}

\author{
F. DELL'ISOLA and A. ROMANO \\ Dipartimento di Matematica e sue Applicazioni dell'Universita' di Napoli, Via Mezzocannone, \\ 8-80134, Napoli, Italy \\ (Communicated by E. S. SUHUBI)
}

\begin{abstract}
The integral balance laws of continuous systems with an interface are obtained as a limit of balance law relative to suitable three-dimensional continuum. This approach supplies a physical interpretation of the quantities appearing in the balance laws and dodges around the not invariance under Galilean transformations of angular momentum balance.
\end{abstract}

\section{INTRODUCTION}

In the papers on continuous systems with an interface two different approaches are developed according to whether a well-established integral balance law is valid for the whole system (see for instance [1, 2]) or for every material volume [3-6].

The local balance equation deduced using the first of these approaches differs from that obtained in the second one because of the presence of some terms called localization residuals which are usually interpreted as describing non-local interactions. In any case the balance laws of mass, linear and angular momentum, energy and entropy are deduced from the aforesaid equation specifying everytime the quantities to be substituted in it.

The aim of this paper is to overcome one problem arising because of the not general validity of these specifications: in fact we observe that, when the interface is nonmaterial, the usual identifications of the quantities appearing in the general balance law in order to obtain the angular momentum balance lead to a relation which is not invariant under Galliean transformations. $\ddagger$

In order to obtain (Section 4) an identification of the quantities appearing in the thermomechanical laws as well as of their transport velocities valid in the more general case in Section 3 we regard the integral balance law of a system with an interface exhibited in Section 2 as a limit of a balance law relative to a suitable threedimensional continuum.

The assumptions made in Section 2 which allow us to perform quoted limiting process find a firm physical justification in experimental evidence (see for instance [7]).

Local thermomechanical balance equations we finally obtain are suitable to describe also those systems in which the interface is nonmaterial. In fact they are always independent of the coordinates adopted on it.

We conclude noting that:

(i) the limiting process we propose allows us to give a more clear physical interpretation to all those surface fields whose introduction was necessary in order to describe nonmaterial interfaces (see [5]).

(ii) our reasonings never used to concept of "material" volume. In fact it turns out to be ambiguous when a part of the interface is included in it and mass transfer occurs between the interface and the surrounding bulk material (for a detailed discussion about this question see [8]).

† Work supported by G.N.F.M. of the Italian C.N.R.

$¥$ If the interface is $p$-material, according to [5] p. 95 , the local equations for angular momentum derived in $[1,5,6]$ are correct. 


\section{GLOBAL BALANCE EQUATION FOR A CONTINUUM WITH AN INTERFACE AND ITS LOCAL FORMULATION}

Let us consider a threedimensional continuum with an interface, occupying at the time $t$ the subset $C(t) \subset R^{3}$ whose boundary $\partial C(t)$ is a regular surface. The interface, carrying own fields, is represented by a family of regular surfaces $S(t) \subset C(t)$, sharing $C(t)$ into two regions $C_{i}(t), i=1,2$. In this section $f, R, \boldsymbol{\Phi}$ will denote volume fields and $f_{\sigma}, R_{\sigma}, \boldsymbol{\Phi}_{\sigma}$ surface fields regular on $C_{i}^{0}$ and on $S$ respectively. Moreover $f, R$, and $\boldsymbol{\Phi}$ are supposed to possess together with their derivatives, limits $f^{ \pm}, R^{ \pm}, \boldsymbol{\Phi}^{ \pm}$at both sides of the surface $S$, which are continuous functions on $S$ itself.

The property represented by the field $f$ is carried by the velocity field $\dot{\mathbf{x}}$, while the one represented by $f_{\sigma}$ is carried along the surface by a tangential velocity $\mathbf{v}_{\tau}$. If we define the $u^{\alpha}(t)$ parameters as coordinates on $S(t), f_{\sigma}, \Phi_{\sigma}$ and $R_{\sigma}$ will become functions of $u^{\alpha}$ and $t$.

We recall the following global integral balance equation (see, for instance $[1,2,8]$ ):

$$
\frac{\mathrm{d}}{\mathrm{d} t}\left(\int_{C(t)} f \mathrm{~d} c+\int_{S(t)} f_{\sigma} \mathrm{d} \sigma\right)=\int_{C(t)} R \mathrm{~d} c+\int_{\partial C(t)} \boldsymbol{\Phi} \cdot \mathbf{N} \mathrm{d} s+\int_{S(t)} R_{\sigma} \mathrm{d} \sigma+\int_{\partial S(t)} \boldsymbol{\Phi}_{\sigma} \cdot \boldsymbol{v} \mathrm{d} l
$$

where $\mathbf{N}$ is the outward, unitary normal vector to $\partial C(t)$, and $\mathbf{v}$ is the outward unitary normal vector to $\partial S(t)$ in the tangent plane to $S(t)$.

As it is well-known, under the aforementioned hypotheses, the previous integral equation is equivalent to the following local ones: $\dagger$

$$
\left.\begin{array}{l}
\frac{\partial f}{\partial t}+\operatorname{div}(f \dot{\mathbf{x}}-\mathbf{\Phi})-R=r \quad \text { in } C_{1}^{0}(t) \cup C_{2}^{0}(t) \\
\frac{\delta_{n}}{\delta t} f_{\sigma}-2 H c_{n} f_{\sigma}-R_{\sigma}-\operatorname{div}_{S}\left(\boldsymbol{\Phi}_{\sigma}-f_{\sigma} \mathbf{v}_{\tau}\right)+\llbracket f(\dot{\mathbf{x}}-\mathbf{c})-\mathbf{\Phi} \rrbracket \cdot \mathbf{n}=s \quad \text { on } S
\end{array}\right\}
$$

where the scalar fields $r$ and $s$, called volume and surface localization residuals respectively, satisfy the conditions:

$$
\int_{C(t)} r \mathrm{~d} c=0, \quad \int_{S(t)} s \mathrm{~d} \sigma=0
$$

The fields $r$ and $s$ are not univocally defined by conditions (2.3), and express non-local interactions. Their introduction can lead to more elaborate theories. In the following they will be assumed vanishing.

In formula (2.2) $\delta_{n} / \delta t$ denotes Thomas' derivative, $H$ and $c_{n}$ are, respectively, the mean curvature and the geometrical normal velocity at any point of $S, \mathbf{n}$ is an unitary vector normal to $S$, and the symbol $\llbracket g \rrbracket$ represents the jump of the volume function $g$ across $S$, whose sign is determined by $\mathbf{n}$.

Formula $(2.2)_{2}$, in the generic $u^{a}$ coordinates, reads:

$$
\frac{\partial \bar{f}_{\sigma}}{\partial t}-\tilde{f}_{\sigma, \alpha} c^{\alpha}-2 H c_{n} \bar{f}_{\sigma}-R_{\sigma}-\Phi_{\sigma, \alpha}^{\alpha}+\left(\tilde{f}_{\sigma} v^{\alpha}\right)_{\mid \alpha}+\llbracket f(\dot{\mathbf{x}}-\mathbf{c})-\mathbf{\Phi} \rrbracket \cdot \mathbf{n}=s \quad \text { on } S
$$

where

$$
\bar{f}_{\sigma}\left(u^{\alpha}, t\right) \equiv f_{\sigma}\left(\mathbf{r}\left(u^{\alpha}, t\right), t\right),\left.\quad \mathbf{c} \equiv \frac{\partial \mathbf{r}}{\partial t}\right|_{u^{\alpha}=\text { const. }} \equiv c^{\alpha} \mathbf{a}_{\alpha}+\left(\mathbf{c}_{n} \cdot \mathbf{n}\right) \mathbf{n}
$$

$\mathbf{r}\left(u_{\alpha}, t\right)$ is the parametric equation of $S(t), \mathbf{a}_{\alpha} \equiv \mathbf{r}_{. \alpha}$ and finally $\Phi_{\sigma \mid \alpha}^{\alpha}$ represents $\operatorname{div}_{S} \boldsymbol{\Phi}$ in the chosen system of coordinates.

\footnotetext{
$\doteqdot$ See for instance $[1,2,5,6]$.
} 


\section{SURFACE QUANTITIES APPEARING IN FORMULA (2.2)}

REGARDED AS LIMITS OF SUITABLE VOLUME ONES

To suggest a coherent identification of the quantities to be substituted in (2.2), when applied in order to obtain all thermomechanical balance laws, we regard it as the result of a suitable limiting process, which starts from the corresponding balance law for threedimensional continua.

More precisely we will consider our system as constituted by just one material, described by fields suffering always continuous but sharp changes in a narrow threedimensional volume $\Delta V(t)$ (whose thickness is $l(t)$ ). If, for simplicity, we renounce to describe in detail all the phenomena occurring in $\Delta V(t)$ we can model the same $\Delta V$ with a family of surfaces $S(t)$ and then define the surfacial distributions on $S(t)$ as averages on $\Delta V$ of the corresponding volume densities. Let us consider, therefore, a threedimensional continuum $C$, occupying, at the time $t$, the region $C(t)$, and suppose that the following balance equation holds:

$$
\frac{\mathrm{d}}{\mathrm{d} t} \int_{C(t)} f \mathrm{~d} c=\int_{C(t)} R \mathrm{~d} c+\int_{\partial C(t)} \boldsymbol{\Phi} \cdot \mathbf{N} \mathrm{d} S .
$$

where $R$ is the volume production of the quantity $f$, and $\mathbf{\Phi} \cdot \mathbf{N}$ is equal to an external given flux of the same property.

The previous considerations lead us to divide the volume $C(t)$ into the regions $C_{1}(t)$, $C_{2}(t)$ and $\Delta V(t)$. For the sake of simplicity, we will make some physically reasonable assumptions about the motion of the considered system:

(a) the volume $\Delta V(t)$ is bounded by two parallel surfaces $S_{i}(t)$, both moving with the same normal velocity $c_{n}$, and by the surface $\Omega$, belonging to $\partial C$. The distance $l$ between the surfaces $S_{i}(t)$ is independent of the time $t$.

(b) The surface $\Omega$ is perpendicular to both $S_{i}$.

We can write each term in (3.1) as the sum of the integrals over the three regions $C_{i}$ and $\Delta V$ (or part of their boundaries), and apply Fubini's theorem to the ones performed over $\Delta V$, where $\Delta V$ is expressed as:

$$
\Delta V(t)=\bigcup_{\mathbf{r} \in S(t)} I(\mathbf{r})
$$

Here $S(t)$ is a surface equidistant from $S_{i}(t)$ whose unitary normal is $\mathbf{n}$, while $I(\mathbf{r}) \equiv\{\mathbf{r}+\xi \mathbf{n},-1 / 2 \leq \xi \leq 1 / 2\}$.

If we introduce the notation:

$$
f_{\sigma l}(\mathbf{r}, t) \equiv \int_{I(\mathbf{r})} f J \mathrm{~d} \xi
$$

where

$$
J(\xi) \mathrm{d} \sigma(\mathbf{r})=\mathrm{d} \sigma(\mathbf{r}+\xi \mathbf{n}) .
$$

the first member of eqn (3.1) becomes:

$$
\frac{\mathrm{d}}{\mathrm{d} t} \int_{C_{1}(t) \cup C_{2}(t)} f \mathrm{~d} c+\frac{\mathrm{d}}{\mathrm{d} t} \int_{S(t)} f_{\sigma l} \mathrm{~d} \sigma .
$$

In the same way, the first term in the second member of (3.1) becomes:

$$
\int_{C_{1}(t) \cup C_{2}(t)} R \mathrm{~d} c+\int_{S(t)} R_{\sigma l} \mathrm{~d} \sigma
$$

With regard to the flux term, let us partition $\partial C(t)$ into its intersections $\partial^{\prime} C(t)$ and $\Omega_{1}$ with $C_{i}(t)$ and $\Delta V(t)$, respectively.

It results in

$$
\Omega_{1}=\bigcup_{\mathbf{r} \in \partial S(t)} I(\mathbf{r})
$$


Moreover let us defined at every point $\mathbf{r} \in S(t)$ and for every tangent vector $\boldsymbol{v}$ to $S$ the surface field:

$$
\boldsymbol{\Phi}_{\sigma l}(\mathbf{r}, \boldsymbol{v}) \equiv \int_{l(\mathbf{r})} \boldsymbol{\Phi}(\mathbf{r}+\xi \mathbf{n}) J^{\prime}(\xi, \mathbf{r}, \mathbf{v}) \mathrm{d} \xi
$$

where $J^{\prime}(\xi, \mathbf{r}, \boldsymbol{v}) \mathrm{d} l(\mathbf{r}, \boldsymbol{v})=\mathrm{d} l\left(\mathbf{r}+\xi_{\mathbf{n}}, \boldsymbol{v}\right) \mathrm{d} l(\mathbf{r}, \boldsymbol{v})$ being the line element of the curve included in $S$, passing through $\mathbf{r}$, which is normal to $\boldsymbol{v}$. A further application of Fubini's theorem leads us to:

$$
\int_{\Omega_{1}} \boldsymbol{\Phi} \cdot \mathbf{N} \mathrm{d} s=\int_{\partial S} \boldsymbol{\Phi}_{\sigma l} \cdot v \mathrm{~d} l .
$$

We remark that the just defined vector field $\boldsymbol{\Phi}_{a l}$ depends on the normal $\boldsymbol{v}$ when $l \neq 0$, so that Gauss theorem is appliable to the second member of eqn (3.4) only in the limit $l \rightarrow 0$ (in fact when $l \rightarrow 0 J^{\prime} \rightarrow 1$ so that the limit $\boldsymbol{\Phi}_{\sigma}$ results independent of $\boldsymbol{v}$ ).

We have now to substitute formulas $(3.2),(3.3),(3.4)$ in formula (3.1) so that it reads now:

$$
\begin{aligned}
\frac{\mathrm{d}}{\mathrm{d} t} \int_{C_{1}(t) \cup C_{2}(t)} f \mathrm{~d} c+\frac{\mathrm{d}}{\mathrm{d} t} \int_{S(t)} f_{\sigma l} \mathrm{~d} \sigma & =\int_{\partial S(t)} \boldsymbol{\Phi}_{\sigma l} \cdot \boldsymbol{v} \mathrm{d} l+\int_{\partial C^{\prime}(t)} \boldsymbol{\Phi} \cdot \mathbf{N} \mathrm{d} \sigma \\
& +\int_{C_{1}(t) \cup C_{2}(t)} R \mathrm{~d} c+\int_{S(t)} R_{\sigma l} \mathrm{~d} \sigma
\end{aligned}
$$

It has to be remarked that in the previous formula both $C_{i}$ and $\partial C^{\prime}$ are regions depending on the parameter $l$. In order to calculate the limit of formula (3.5) we use a well-known derivation formula (see for instance $[8,9]$ ) and a simple application of change-of -variable theorem in integration theory. In this way we derive:

$$
\frac{\mathrm{d}}{\mathrm{d} t} \int_{C_{1} \cup C_{2}} f=\int_{C_{1} \cup C_{2}}\left(\frac{\partial f}{\partial t}+\operatorname{div} f \dot{\mathbf{x}}\right)+\int_{S}\left\{f_{2}\left(\dot{x}_{n_{2}}-c_{n_{2}}\right) J_{2}-f_{1}\left(x_{n_{1}}-c_{n_{1}}\right) J_{1}\right\} \mathrm{d} \sigma
$$

where $f_{i}$ is the value assumed by the function $f$ on the surface $S_{i}, c_{n_{i}}$ is the normal speed of the same surface, $J_{i} \equiv \mathrm{d} \sigma_{i} / \mathrm{d} \sigma$ being $\mathrm{d} \sigma_{i}$ and $\mathrm{d} \sigma$ the surface elements on the surfaces $S_{i}$ and $S$ respectively, $\dot{x}_{n_{i}} \equiv \dot{\mathbf{x}}_{i} \cdot \mathbf{N}_{i}$ where $\mathbf{N}_{i}$ is the normal to $S_{i}$ and $\dot{\mathbf{x}}_{i}$ is the value of the field $\dot{\mathbf{x}}$ on the same surface.

In order to calculate the second term of the first member in formula (3.5) we have to define $\left[\right.$ see $\left.(2.2)_{2}\right]$ the velocity $\mathbf{v}_{\tau l}$ which "carries" $f_{o l}$ along $S$ in terms of $\dot{\mathbf{x}}$ carrying $f$. To this aim we will assume that:

$$
\mathbf{v}_{l}(\mathbf{r}) \equiv \frac{\int_{I(\mathbf{r})} f \dot{\mathbf{x}} J \mathrm{~d} \xi}{\int_{I(\mathbf{r})} f J \mathrm{~d} \xi} .
$$

This hypothesis assures that when $f$ is identified with $\rho$ (mass density) $\mathbf{v}_{l}(\mathbf{r})$ becomes the velocity of the centre of mass of the material particle laying in $\Delta V(t)$ (see Section 3 too). Using the definition of Thomas' derivative (see for instance [8]), we obtain:

$$
\frac{\mathrm{d}}{\mathrm{d} t} \int_{S(t)} f_{\sigma l}=\int_{S(t)} \frac{\delta_{n}}{\delta t} f_{\sigma l}-2 H c_{n} f_{\sigma l}+\int_{\partial S(t)} f_{\sigma l} \mathbf{v}_{\tau l} \cdot \mathbf{v} \mathrm{d} l
$$

where Gauss' divergence Theorem was applied.

Substituting formulas (3.6) and (3.8) in (3.5), calculating the limit for $l \rightarrow 0$, $\dagger$ and recalling again Gauss' divergence Theorem we obtain an integral balance equation in

$\dagger$ We obviously suppose that for all the families of functions $f_{\sigma l}, R_{\sigma l}, \Phi_{\sigma l}, v_{l}$ there exists the limit (in a norm which allows the inversion of the limit with the integrals appearing in formula (3.5)) when $l \rightarrow 0$. 
Table 1

\begin{tabular}{cc}
\hline $\begin{array}{c}\text { Quantity appearing in } \\
\text { formula }(2.2)_{2}\end{array}$ & As determined in function of limit quantities \\
\hline$f_{\sigma}$ & $f_{\sigma}=\lim _{l \rightarrow 0} f_{\sigma l}$ \\
$\boldsymbol{\Phi}_{\sigma}$ & $\boldsymbol{\Phi}_{\sigma}=\lim _{l \rightarrow 0} \boldsymbol{\Phi}_{\sigma l}$ \\
$R_{\sigma}$ & $\frac{\delta_{g t}}{\delta t} f_{\sigma}-\lim _{t \rightarrow 0}\left(\frac{\delta_{n}}{\delta t} f_{\sigma t}\right)+\lim _{t \rightarrow 0} R_{\sigma t}$ \\
\hline
\end{tabular}

the form:

$$
\begin{aligned}
\int_{C(t)}\left(\frac{\partial f}{\partial t}+\operatorname{div} f \dot{\mathbf{x}}\right) \mathrm{d} c+\int_{S(t)} \llbracket f(\dot{\mathbf{x}}-\mathbf{c}) \cdot \mathbf{n} \rrbracket \mathrm{d} \sigma \\
+\lim _{l \rightarrow 0} \int_{S(t)} \frac{\delta_{n}}{\delta t} f_{\sigma l} \mathrm{~d} \sigma+\int_{S(t)}\left[-2 H c_{n} f_{\sigma}+\operatorname{div}_{S}\left(f_{\sigma} \mathbf{v}_{\tau}\right)\right] \mathrm{d} \sigma \\
\quad=\int_{\partial C(t)} \boldsymbol{\Phi} \cdot \mathbf{N} \mathrm{d} \sigma+\int_{\partial S(t)} \boldsymbol{\Phi}_{\sigma} \cdot \mathbf{v} \mathrm{d} l+\int_{C(t)} R \mathrm{~d} c+\int_{S} \hat{R}_{\sigma} \mathrm{d} c
\end{aligned}
$$

where

$$
\lim _{l \rightarrow 0} R_{\sigma l}=\hat{R}_{\sigma} .
$$

Formula (3.9) leads us to the following local formula:

$$
\lim _{l \rightarrow 0}\left(\frac{\delta_{n}}{\delta t} f_{\sigma l}\right)+\operatorname{div}_{S}\left(f_{\sigma} \mathbf{v}_{\tau}\right)-2 H c_{n} f_{\sigma}-\operatorname{div}_{S} \boldsymbol{\Phi}_{\sigma}+\llbracket f(\dot{\mathbf{x}}-\mathbf{c})-\boldsymbol{\Phi} \rrbracket \cdot \mathbf{n}-\hat{R}_{\sigma}=0
$$

which can be written as follows:

$$
\begin{aligned}
& \frac{\delta_{n}}{\delta t} f_{\sigma}+\operatorname{div}_{S}\left(f_{\sigma} \mathbf{v}_{\tau}\right)-2 H c_{n} f_{\sigma}-\operatorname{div}_{S} \boldsymbol{\Phi}_{\sigma}+\llbracket f(\dot{\mathbf{x}}-\mathbf{c})-\boldsymbol{\Phi} \rrbracket \cdot \mathbf{n}+ \\
&-\hat{R}_{\sigma}+\left\{\lim _{l \rightarrow 0} \frac{\delta_{n}}{\delta t} f_{\sigma l}-\frac{\delta_{n}}{\delta t} f_{\sigma}\right\}=0 .
\end{aligned}
$$

By comparing (3.10) and $(2.2)_{2}$ we are led to the identifications listed in Table 1.

Table 1 will be used in the following section.

Obviously, the previous derivation has just an heuristic value, as it is clear that both the assumptions that a threedimensional scheme can describe the physical situation in consideration, and the assumptions about the motion of the region $\Delta V$ could not be generally valid.

\section{LOCAL BALANCE EQUATIONS FOR THERMOMECHANICAL SYSTEMS WITH INTERFACES}

In this section in addition to the hypotheses (a), (b) of Section 3, we suppose that:

(c) the tangential velocity field $\mathbf{v}_{\tau}$ (carrying mass density) is constant along the interval $I(\mathbf{r})$ for every $\mathbf{r} \in S$, and independent of $l$.

This implies that:

$$
\dot{\mathbf{x}}_{\tau}^{+}=\dot{\mathbf{x}}_{\tau}^{-}=\mathbf{v}_{\tau} .
$$

Condition c) can be regarded as a consequence of the viscosity of the considered threedimensional continuum in the neighbourhood of the interface.

In order to obtain the local form of balance relations for a thermomechanical system with interfaces, we assign the quantities appearing in formula $(2.2)_{2}$ according to Table 1. 
Table 2

\begin{tabular}{lccc}
\hline & $f$ & $f_{\sigma l}$ & $f_{\sigma}$ \\
\hline Mass density & $\rho$ & $\rho_{\sigma l} \equiv \int_{I} \rho J \mathrm{~d} \xi$ & $\rho_{\sigma}$ \\
Linear mom. density & $\rho \dot{x}^{i}$ & $P_{\sigma l}^{i} \equiv \int_{I} \rho \dot{x}^{i} J \mathrm{~d} \xi$ & $\rho_{\sigma} v^{i}$ \\
Angular mom. density & $\varepsilon^{i j h} x^{j} \rho \dot{x}^{h}$ & $M_{\sigma l}^{i} \equiv \int_{I} \varepsilon^{i j h} x^{j} \rho \dot{x}^{h} J \mathrm{~d} \xi$ & $\rho_{\sigma} \varepsilon^{i j h} \dot{r}^{\prime} \tau^{h}$ \\
Energy density & $\rho\left(\frac{1}{2} \dot{\mathrm{x}}^{2}+\varepsilon\right)$ & $E_{\sigma l} \equiv \int_{I} \rho\left(\frac{1}{2} \dot{\mathrm{x}}^{2}+\varepsilon\right) J \mathrm{~d} \xi$ & $\rho_{\sigma}\left(\frac{1}{2} v^{2}+\varepsilon_{\sigma}\right)$ \\
Entropy density & $\rho \eta$ & $H_{\sigma l} \equiv \int_{I} \rho \eta \eta \mathrm{d} \xi$ & $\rho_{\sigma} \eta_{\sigma}$ \\
\hline
\end{tabular}

We begin by evaluating the limits:

$$
\lim _{l \rightarrow 0} f_{\sigma l} \equiv f_{\sigma}
$$

when $f$ and correspondently $f_{\sigma l}$ are given by Table 2 , where the integration interval $I$ is in all cases a function of the point $\mathbf{r}$ on the surface $S$.

In Table 2 we have defined the surface mass density $\rho_{\sigma}$ as the limit

$$
\lim _{l \rightarrow 0} \rho_{\sigma l} \equiv \rho_{\sigma} .
$$

With regard to the surface linear momentum density $\mathbf{P}_{\sigma l}$, we remark that, by virtue of mean value theorem it can be represented in the form $\mathbf{P}_{\sigma l} \equiv \rho_{\sigma l} \mathbf{v}_{l}$, where according to $(3.7)$ :

$$
\mathbf{v}_{l} \equiv \frac{\int_{I(\mathbf{r})} \rho \dot{\mathbf{x}} J \mathrm{~d} \xi}{\int_{I(\mathbf{r})} \rho J \mathrm{~d} \xi}
$$

is the velocity of the center of mass of the particles instantaneously laying in $\mathrm{d} \sigma \times I(\mathbf{r})$.

Owing to (4.2) we have:

$$
\mathbf{P}_{\sigma} \equiv \lim _{l \rightarrow 0} \mathbf{P}_{\sigma l}=\rho_{\sigma} \mathbf{v} .
$$

It is worthwhile to observe that hypothesis c) implies the tangential component $\mathbf{v}_{\tau l}$ of $\mathbf{v}_{l}$ to be independent of $l$ and $\rho$ :

$$
\mathbf{v}_{\tau}=\mathbf{v}_{l \tau}, \quad \forall l>0 .
$$

Let us now use definition (4.2) in order to represent

$$
\lim _{l \rightarrow 0} E_{\sigma l}
$$

in the usual form:

$$
\frac{1}{2} \rho_{\sigma} \mathbf{v}^{2}+\rho_{\sigma} \varepsilon_{\sigma}
$$

where we have defined

$$
\lim _{l \rightarrow 0} \int_{I(l)}\left(\rho \varepsilon+\frac{1}{2} \rho \dot{x}^{\prime 2}\right) J \mathrm{~d} \xi \equiv \rho_{\sigma} \varepsilon_{\sigma} .
$$

This is easily done when we observe that

$$
\dot{\mathbf{x}}=\mathbf{v}+\dot{\mathbf{x}}^{\prime},
$$


being $\dot{\mathbf{x}}^{\prime}$ the velocity of the particle in $\Delta V$ with respect to the mass center frame and recall that linear momentum in this frame vanishes.

Concerning last row of Table 2 , it is obvious that we can assume that:

$$
\lim _{l \rightarrow 0} H_{\sigma l}=\rho_{\sigma} \eta_{\sigma}
$$

We have already seen that surface fields defined with our limiting process are not completely independent. This fact becomes even more evident when we finally look for the limit:

$$
\lim _{l \rightarrow 0} \mathbf{M}_{\sigma l}
$$

To this aim let us express angular momentum surface density $\mathbf{M}_{\sigma l}$ in the following way:

$$
\mathbf{M}_{\sigma l}=\int_{I}\left(\mathbf{r} \times x^{\prime} \mathbf{n}\right) \times \rho \dot{\mathbf{x}} \mathrm{d} x^{\prime} .
$$

Here $\mathbf{r}$ is the position of the point on $S$, while $x^{\prime} \mathbf{n}=\mathbf{x}-\mathbf{r}$.

With simple algebra, we deduce:

$$
\mathbf{M}_{\sigma l}=\mathbf{r} \times \mathbf{P}_{\sigma l}+\mathbf{n} \times \int_{I} x^{\prime} \rho \dot{\mathbf{x}}_{\tau} \mathrm{d} x^{\prime} .
$$

Recalling again hypothesis c) we obtain:

$$
\mathbf{M}_{\sigma l}=\mathbf{r} \times \mathbf{P}_{\sigma l}+\rho_{\sigma} d_{l} \mathbf{n} \times \mathbf{v}_{\tau}
$$

where $d_{l}$ represents the distance between $S$ and the center of mass of the particle instantaneously laying in $d \sigma \times I(\mathbf{r})$ along the normal to $S$. From this equation, we have:

$$
\lim _{l \rightarrow 0} \mathbf{M}_{\sigma l}=\mathbf{r} \times \rho_{\sigma} \mathbf{v} \equiv \mathbf{M}_{\sigma}
$$

In order to perform the substitutions listed in Table 1 we have to calculate the difference:

$$
\frac{\delta_{n}}{\delta t} f_{\sigma}-\lim _{l \rightarrow 0} \frac{\delta_{n}}{\delta t} f_{\sigma l}
$$

when $f_{\sigma l}, f_{\sigma}$ are identified everytime with $\rho_{\sigma l}, \rho_{\sigma} ; P_{\sigma l}^{i}, P_{\sigma}^{i} ; M_{\sigma l}^{i}, M_{\sigma}^{i} ; E_{\sigma l}, E_{\sigma} ; H_{\sigma l}, H_{\sigma}$.

It is obvious that differences (4.6) will be greatly influenced by the modalities with which the limits when $l \rightarrow 0$ are attained. When it is possible to assume:

(d) $\forall l$ suitably small $f_{\sigma l}=f_{\sigma}$, it clearly results that this difference vanishes.

We observe now that it is possible to assume hypothesis d) valid when the quantity $f$ in Table 1 is taken equal respectively to mass, linear momentum, energy and entropy.

This statement can be justified from a physical viewpoint when we remark that is possiblet to assume that the phenomenon our theory is modelling (the formation of the interface) cannot take place without involving a finite quantity of matter (or linear momentum, or energy, or entropy) quantity which will always fill (or be carried by) the volume $\Delta V$, independently of its thickness $l$ (experimental evidences supporting this assumption can be found for instance in [7]). It is obvious that hypothesis d) implies the difference (4.6) is vanishing for all quoted quantities, whereas for the angular momentum, owing to (4.4) and (4.5), we obtain:

$$
\frac{\delta_{n}}{\delta t} \mathbf{M}_{\sigma}-\lim _{l \rightarrow 0}\left(\frac{\delta_{n}}{\delta t} \mathbf{M}_{\sigma t}\right)=\rho_{\sigma} \mathbf{n} \times \mathbf{v}_{\tau}\left(\lim _{l \rightarrow 0} \frac{\delta_{n}}{\delta t} d_{l}\right) .
$$

The limit appearing in second member of eqn (4.7) can be easily calculated when we recall that $d_{l}$ represents the distance (along the normal to $S$ ) between $S$ and the center

+ We do not want to imply that more sophisticated theories cannot be developed where quoted hypotheses are completely or partially given up. What we need to underline now is that we can coherently assume them. 


\begin{tabular}{|c|c|c|c|c|c|}
\hline Balance of $\rightarrow$ & Mass & Lin. mom. & Angular mom. & Energy & Entropy \\
\hline$f \rightarrow$ & $\rho$ & $\rho \dot{x}^{i}$ & $\rho \varepsilon_{j h}^{i} x^{j} \dot{x}^{h}$ & $\rho\left(\frac{1}{2} \mathbf{v}^{2}+\varepsilon\right)$ & $\rho \eta$ \\
\hline$f_{o} \rightarrow$ & $\rho_{o}$ & $\rho_{\sigma} v^{i}$ & $\rho_{\sigma} \varepsilon_{j h}^{i} r^{j} v^{h}$ & $\rho_{\sigma}\left(\frac{1}{2} v^{2}+\varepsilon_{\sigma}\right)$ & $\rho_{\sigma} \eta_{\sigma}$ \\
\hline$\Phi \rightarrow$ & 0 & $\mathbf{T}^{\prime}$ & $\varepsilon_{j, i}^{i} x^{j} T^{h k}$ & $\dot{\mathbf{x}} \cdot \mathbf{T}-\mathbf{h}$ & $-h / \theta$ \\
\hline $\begin{array}{c}\Phi_{\mathrm{\sigma}} \rightarrow \\
R \rightarrow\end{array}$ & $\begin{array}{l}0 \\
0\end{array}$ & $\begin{array}{l}\mathbf{T}_{o}^{j} \\
\rho b^{i}\end{array}$ & $\begin{array}{l}\varepsilon_{j h}^{i} \gamma^{j} T_{j}^{h \alpha} \\
\rho \varepsilon_{j h}^{i} x^{\prime} b^{h}\end{array}$ & $\begin{array}{c}\boldsymbol{v} \cdot \mathbf{T}_{\sigma}-\mathbf{h}_{o} \\
\rho r\end{array}$ & $\begin{array}{c}-\mathrm{h}_{\sigma} / \theta_{\sigma} \\
\rho r / \theta\end{array}$ \\
\hline$R_{\sigma} \rightarrow$ & 0 & $\rho_{\sigma} b_{\sigma}^{i}$ & $\left.\begin{array}{c}\rho_{\sigma} \varepsilon_{j h}^{i} r^{j} b_{\sigma}^{h} \\
+ \\
(v-c)_{n} \rho_{\sigma} \varepsilon_{j h}^{i} n^{j} v^{h}\end{array}\right\}$ & $\rho_{\sigma} r_{\sigma}$ & $\rho_{\sigma} r_{\sigma} / \theta_{\sigma}$ \\
\hline
\end{tabular}

of mass of the particles instantaneously laying in $d \sigma \times I(\mathbf{r})$.

$$
\lim _{l \rightarrow 0} \frac{\delta_{n} d_{l}}{\delta t}=v_{n}-c_{n}
$$

We note that this limit is equal to zero when and only when $v_{n}=c_{n}$, so that it plays a relevant role in the theory we are developing where this equality is not satisfied. On the other hand when we describe surface phenomena occurring during phase transition (see also [10]), it could be necessary to suppose $c_{n} \neq v_{n}$, as it has been already remarked in Kosinski [5].

We resume the previous results in Table 3 , where: $\rho$ is volume mass density, $\rho_{\sigma}$ is surface mass density, $\dot{\mathbf{x}}$ is the velocity field in threedimensional continua $C_{i}, \mathbf{v}$ is the surface velocity field, $\mathbf{T}$ is the Cauchy stress tensor in $C_{i}, \mathbf{T}_{o}$ is the surface stress tensor, whose tangential components are $T_{\sigma}^{i \alpha}$, (vectorial notation $\mathbf{T}_{\sigma}^{i}$ obviously denoting the vector $T^{i \mathbf{a}_{\alpha}}$ where $\mathbf{a}_{\alpha}$ is a set of vectors constituting a tangent base on $\left.S(t)\right) \rho \mathbf{b}$ and $\rho_{\sigma} \mathbf{b}_{\sigma}$ are given volume and surface force density, $\varepsilon$ is mass internal energy density in $C_{i}$, $\varepsilon_{\sigma}$ is surface mass internal energy density, $\mathbf{h}$ and $\mathbf{h}_{\sigma}$ are volume and surface heat fluxes, $\eta$ and $\eta \sigma$ are volume and surface mass entropy densities, and finally $r$ and $r_{\sigma}$ are mass heat production density in $C_{i}$ and on $S$.

From Table 3 we derive the following local balance equations and jump conditions:

$$
\begin{gathered}
\frac{\partial \rho}{\partial t}+\operatorname{div} \rho \dot{\mathbf{x}}=0 ; \quad \rho \ddot{\mathbf{x}}=\operatorname{div} \mathbf{T}+\rho \mathbf{b} ; \quad \mathbf{T}=\mathbf{T}^{T} \\
\rho \dot{\varepsilon}=\mathbf{T}: \operatorname{grad} \dot{\mathbf{x}}-\operatorname{div} \mathbf{h}+\rho r ; \quad \rho \dot{\eta} \geq-\operatorname{div}(\mathbf{h} / \theta)+\rho r / \theta
\end{gathered}
$$

in $C_{1}^{0} \cup C_{2}^{0}$, whereas on $S$ it results:

$$
\begin{aligned}
& \frac{\partial \rho_{\sigma}}{\partial t}+\left(\rho_{\sigma} V^{\alpha}\right)_{\mid \alpha}+\rho_{\sigma} \eta_{\alpha}^{\alpha}+\left(\rho_{\sigma} v^{\alpha}\right)_{\mid \alpha}+\llbracket \rho(\dot{\mathbf{x}}-\mathbf{c}) \rrbracket \cdot \mathbf{n}=0 \\
& \frac{\partial}{\partial t}\left(\rho_{\sigma} \mathbf{v}\right)+\rho_{\sigma} \mathbf{v} \eta_{\alpha}^{\alpha}+\left(\rho_{\sigma} \mathbf{v} V^{\alpha}+\rho_{\sigma} \mathbf{v} v^{\alpha}-\mathbf{T}_{\sigma}^{(\alpha)}\right)_{\mid \alpha}+\llbracket \rho \dot{\mathbf{x}} \otimes(\dot{\mathbf{x}}-\mathbf{c})-\mathbf{T} \rrbracket \cdot \mathbf{n}=\mathbf{0} \\
& \frac{\partial}{\partial t}\left(\rho_{\sigma} \mathbf{r} \times \mathbf{v}\right)+\left(\rho_{\sigma} \mathbf{r} \times \mathbf{v} V^{\alpha}\right)_{\mid \alpha}+\rho_{\sigma} \eta_{\alpha}^{\alpha} \mathbf{r} \times \mathbf{v}+\left(\rho_{\sigma} \mathbf{r} \times \mathbf{v} v^{\alpha}-\mathbf{r} \times \mathbf{T}_{\sigma}^{(\alpha)}\right)_{\mid \alpha} \\
& \quad+\rho_{\sigma}\left(v_{n}-c_{n}\right) \mathbf{n} \times \mathbf{v}_{\tau}+\llbracket \rho \mathbf{x} \times \dot{\mathbf{x}} \otimes(\dot{\mathbf{x}}-\mathbf{c})-\mathbf{x} \times \mathbf{T} \rrbracket \cdot \mathbf{n}=\mathbf{0} \\
& \frac{\partial}{\partial t}\left(\rho_{\sigma}\left(\frac{1}{2} \mathbf{v}^{2}+\varepsilon_{\sigma}\right)\right)+\left(\rho_{\sigma}\left(\frac{1}{2} \mathbf{v}^{2}+\varepsilon_{\sigma}\right) V^{\alpha}\right)_{\mid \alpha}+\rho_{\sigma} \eta_{\alpha}^{\alpha}\left(\frac{1}{2} \mathbf{v}^{2}+\varepsilon_{\sigma}\right)-\left(\mathbf{v} \cdot \mathbf{T}_{\sigma}^{(\alpha)}\right)_{\mid \alpha} \\
& \quad+\left(\rho_{\sigma}\left(\frac{1}{2} \mathbf{v}^{2}+\varepsilon_{\sigma}\right) v^{\alpha}\right)_{\mid \alpha}+\left(\mathbf{h}_{\sigma}^{(\alpha)}\right)_{\mid \alpha}+\llbracket \rho(\dot{\mathbf{x}}-\mathbf{c})\left(\frac{1}{2} \dot{\mathbf{x}}^{2}+\varepsilon\right)-\dot{\mathbf{x}} \cdot \mathbf{T}+\mathbf{h} \rrbracket \cdot \mathbf{n}=0 \\
& \frac{\partial}{\partial t}\left(\rho_{\sigma} \eta_{\sigma}\right)+\left(\rho_{\sigma} \eta_{\sigma}\left(V^{\alpha}+v^{\alpha}\right)\right)_{\mid \alpha}+\rho_{\sigma} \eta_{\alpha}^{\alpha} \eta_{\sigma}+\left(\frac{\mathbf{h}_{\sigma}}{\theta_{\sigma}}\right)_{\mid \alpha}+\llbracket \rho \eta(\dot{\mathbf{x}}-\sim)+\mathbf{h} / \theta \rrbracket \cdot \mathbf{n} \geq 0
\end{aligned}
$$


In the preceding formulas arbitrary surface coordinates $u^{\alpha}$ have been used, $\eta_{\alpha}^{\alpha} \equiv c_{\mid \alpha}^{\alpha}-$ $2 H c_{n}$, and $V^{\alpha}=-c^{\alpha}$ is Thomas' derivative of $u^{\alpha}$. After collecting in (4.9) $)_{2}$ the terms containing $v$ and substituting in the so obtained expression $(4.9)_{1}$, it results:

$$
\rho_{\sigma} \frac{\delta_{n}}{\delta t} \mathbf{v}+\rho_{\sigma} \nabla_{S} \mathbf{v} \cdot \mathbf{v}_{\tau}-\operatorname{div}_{S} \mathbf{T}_{\sigma}+\llbracket \rho(\dot{\mathbf{x}}-\mathbf{v}) \otimes(\dot{\mathbf{x}}-\mathbf{c})-\mathbf{T} \rrbracket \cdot \mathbf{n}=\mathbf{0}
$$

Similarly, we can collect in $(4.9)_{4}$ the terms where appear the quantities: $\mathbf{v}$ and $\left(\frac{1}{2} \mathbf{v}^{2}+\varepsilon_{\sigma}\right)$ and substitute the expressions obtained from eqns $(4.9)_{1},(4.10)$. This leads to:

$$
\begin{aligned}
\rho_{\sigma} \frac{\delta_{n}}{\delta t} \varepsilon_{\sigma}+\rho_{\sigma} \nabla_{S} \varepsilon_{\sigma} & \cdot \mathbf{v}_{\tau}-\mathbf{T}_{\sigma}: \nabla_{S} \mathbf{v}+\operatorname{div}_{S} \mathbf{h}_{\sigma} \\
& +\llbracket \rho\left(\frac{1}{2}(\dot{\mathbf{x}}-\mathbf{v})^{2}+\left(\varepsilon-\varepsilon_{\sigma}\right)\right)(\dot{\mathbf{x}}-\mathbf{c})-(\dot{\mathbf{x}}-\mathbf{v}) \cdot \mathbf{T}+\mathbf{h} \rrbracket \cdot \mathbf{n}=0 .
\end{aligned}
$$

Finally, we can collect in $(4.9)_{5}$ the terms containing $\theta_{\sigma} \eta_{\sigma}$ and $\theta_{\sigma}$, substitute eqn (4.9) express $\operatorname{div}_{S} \mathbf{h}_{\sigma}$ by means of (4.11) and define $\psi_{\sigma} \equiv \varepsilon_{\sigma}-\theta_{\sigma} \eta_{\sigma}$, thus obtaining:

$$
\begin{aligned}
& -\rho_{\sigma}\left(\psi_{\sigma}^{\prime}+\eta_{\sigma} \theta_{\sigma}^{\prime}\right)+\mathbf{T}_{\sigma}: \nabla_{S} \mathbf{v}-\frac{\mathbf{h}_{\sigma}}{\theta_{\sigma}} \cdot \nabla_{S} \theta_{\sigma}+\psi_{\sigma} \llbracket \rho(\dot{\mathbf{x}}-\mathbf{c}) \rrbracket \cdot \mathbf{n}+ \\
& \quad-\llbracket \rho\left(\frac{1}{2}(\dot{\mathbf{x}}-\mathbf{v})^{2}+\varepsilon-\theta_{\sigma} \eta\right)(\dot{\mathbf{x}}-\mathbf{c})-(\dot{\mathbf{x}}-\mathbf{v}) \cdot \mathbf{T} \rrbracket \cdot \mathbf{n}+\llbracket\left(\frac{\theta_{\sigma}}{\theta}-1\right) \mathbf{h} \rrbracket \cdot \mathbf{n} \geq 0
\end{aligned}
$$

where

$$
\psi_{\sigma}^{\prime} \equiv \frac{\delta_{n}}{\delta t} \psi_{\sigma}+\nabla_{S} \psi_{\sigma} \cdot \mathbf{v}_{\tau} .
$$

In an arbitrary system of coordinates $u^{\alpha}$ on the surface $S$ previous formulas reads:

$$
\begin{gathered}
\rho_{\sigma} \frac{\partial \mathbf{v}}{\partial t}+\rho_{\sigma}\left(v^{\alpha}-c^{\alpha}\right) \mathbf{v}, \alpha-\mathbf{T}_{\sigma \mid \alpha}^{(\alpha)}+\llbracket \rho(\dot{\mathbf{x}}-\mathbf{v}) \otimes(\dot{\mathbf{x}}-\mathbf{c})-\mathbf{T} \rrbracket \cdot \mathbf{n}=\mathbf{0} \\
\rho_{\sigma} \frac{\partial \varepsilon_{\sigma}}{\partial t}+\rho_{\sigma}\left(v^{\alpha}-c^{\alpha}\right) \varepsilon_{\sigma, \alpha}-\mathbf{T}_{\sigma}^{(\alpha)} \cdot \mathbf{v}_{, \alpha}+h_{\sigma \mid \alpha}^{\alpha} \\
\left.+\llbracket \rho\left(\frac{1}{2}(\dot{\mathbf{x}}-\mathbf{v})^{2}+\left(\varepsilon-\varepsilon_{\sigma}\right)\right)(\dot{\mathbf{x}}-\mathbf{c})-(\dot{\mathbf{x}}-\mathbf{v}) \cdot \mathbf{T}+\mathbf{h}\right] \cdot \mathbf{n}=0 \\
-\rho_{\sigma}\left(\psi_{\sigma}^{\prime}+\eta_{\sigma} \theta_{\sigma}^{\prime}\right)+\mathbf{T}_{\sigma}^{(\alpha)} \cdot \mathbf{v}_{, \alpha}-\frac{h_{\sigma}^{\alpha}}{\theta_{\sigma}} \theta_{\sigma, \alpha}+\psi_{\sigma} \llbracket \rho(\dot{\mathbf{x}}-\mathbf{c}) \rrbracket \cdot \mathbf{n} \\
\left.-\left[\rho\left(\frac{1}{2}(\dot{\mathbf{x}}-\mathbf{v})^{2}+\varepsilon-\theta_{\sigma} \eta\right)(\dot{\mathbf{x}}-\mathbf{c})-(\dot{\mathbf{x}}-\mathbf{v}) \cdot \mathbf{T}\right]\right] \cdot \mathbf{n}+\llbracket\left(\frac{\theta_{\sigma}}{\theta}-1\right) \mathbf{h} \rrbracket \cdot \mathbf{n} \geq 0
\end{gathered}
$$

In particular, when $\theta^{+}=\theta^{-}=\theta_{\sigma}$ eqn (4.15) becomes:

$$
\begin{aligned}
-\rho_{\sigma}\left(\psi_{\sigma}^{\prime}+\eta_{\sigma} \theta_{\sigma}^{\prime}\right)+\mathbf{T}_{\sigma}^{(\alpha)} & \cdot \mathbf{v}_{, \alpha}-\frac{h_{\sigma}^{\alpha}}{\theta_{\sigma}} \theta_{\sigma, \alpha}+\psi_{\sigma} \llbracket \rho(\dot{\mathbf{x}}-\mathbf{c}) \rrbracket \cdot \mathbf{n}+ \\
- & -\left[\rho\left\{\left(\frac{1}{2}(\dot{\mathbf{x}}-\mathbf{v})^{2}+\psi\right)(\dot{\mathbf{x}}-\mathbf{c})-(\dot{\mathbf{x}}-\mathbf{v}) \cdot \mathbf{T} / \rho\right\}\right] \cdot \mathbf{n} \geq 0
\end{aligned}
$$

Let's now consider eqn $(4.9)_{3}$.

Collecting once more the terms containing $\mathbf{r}$, remembering that, on $S(t)$, is $\mathbf{x}^{-}=\mathbf{x}^{+}=\mathbf{r}$, and taking into account balance equation for linear momentum, it is easily seen that balance equation for angular momentum is equivalent to the following relation:

$$
\mathbf{r}_{, \alpha} \times \mathbf{T}_{\sigma}^{(\alpha)}=\mathbf{0}
$$


This equation reduces to the following scalar ones:

$$
\begin{aligned}
& T^{\alpha \beta}=T^{\beta \alpha} \\
& T^{\alpha n}=0
\end{aligned}
$$

The first of them expresses the symmetry of the tangential component of the surface stress tensor, which is a well-known result of the classical theories. The second imposes that the interface cannot exert normal stresses.

We conclude emphasizing that the angular momentum balance law assumes a form which is not Galilean invariant if we do not take into account the following source terms appearing in Table 3 and singled out by means of quoted limiting process:

$$
\mathbf{s} \equiv\left(v_{n}-c_{n}\right) \rho_{\sigma} \mathbf{n} \times \mathbf{v} .
$$

In fact it is easily seen that in this case eqn $(4.18)_{2}$ becomes:

$$
T^{\infty n}=s^{\alpha} \text {, }
$$

which obviously is not Galilean invariant because of the presence of the quantity $\mathbf{v}$ in the cross product.

\section{REFERENCES}

[1] W. KOSINSKI, Thermodynamics of singular surfaces and phase transition. In Free Moving Boundary Problems: Applications and Theory, Vol. III (Edited by A. Bossavit, A. Damlamian, M. Fremond). Pitman (1985)

[2] E. S. SUHUBI, Balance laws of continuum physics. Lett. Appl. Engng Sci. 21, 283-288 (1983).

[3] A. ROMANO, Thermodynamics of a continuum with an interface and Gibbs' rule. Ric. di Mat. XXXI (1982).

[4] P. FERGOLA and A. ROMANO, On thermodynamics of fluid and solid phases. Ric. di Mat. II (1983).

[5] W. KOSINSKI, Field Singularities and Wave Analysis in Continuum Mechanics. PWN-Polish Scientific Publisher, Warsaw (1987).

[6] G. P. MOECKEL, Thermodynamics of an interface. Arch. Ratl Mech. Anal. 57 (1975)

(7) J. N. MURREI and E. A. BOUCHER, Properties of Liquids and Solutions. Wiley; Chichester (1982).

[8] F. DELL'ISOLA and A. ROMANO, On a general balance law for continua with an interface (to appear in Ricerche di Matematica, Napoli) (1987).

[9] J. SERRIN, On the uniqueness of compressible fluid motions. Arch. Ratl Mech. Anal. 34, 271-288 (1959).

[10] F. DELL'ISOLA and A. ROMANO, A phenomenological approach to phase transition in classical field theory. Int. J. engng Sci. 25, 1469-1475 (1987). 